\title{
Symbolic, identity, and probe delayed matching of sounds by the bottlenosed dolphin
}

\author{
LOUIS M. HERMAN \\ University of Hawaii, Honolulu, Hawaii 96822 \\ and \\ ROGER K. R. THOMPSON \\ Franklin and Marshall College, Lancaster, Pennsylvania 17604
}

\begin{abstract}
The short-term memory for sounds of the bottlenosed dolphin was tested using symbolic, identity, and probe forms of the delayed matching-to-sample (DMS) task. The forms differed in the number (one or two) or nature (symbolic or identity matches of sample sounds) of postdelay test stimuli available as memory retrieval cues. Although symbolic DMS was difficult to learn, the final performance level was approximately equal to that for identity or probe DMS. On all tasks, the dolphin's responses were above $80 \%$ correct through to delays of $90 \mathrm{sec}$ and, in some cases, through to delays of 180 and $240 \mathrm{sec}$, the "limits" being governed mainly by the dolphin's reluctance to continue being tested at long delays. Encoding of sample stimuli into their learned symbolic representation was hypothesized to have reduced symbolic DMS to a recognition memory task, resulting in the observed equivalence of performance with the other two recognition memory tasks. The probe DMS results, unlike those for identity or symbolic DMS, showed no significant proactive interference effects from samples of prior trials. Instead, proactive interference was traceable to the probe value of the prior trial. Overall, the auditory DMS data for the dolphin were functionally similar to results reported for monkeys tested on symbolic, identity, and probe visual DMS tasks.
\end{abstract}

Studies using variations of the delayed matchingto-sample (DMS) procedure have found the auditory short-term memory of the bottlenosed dolphin Tursiops truncatus to be precise and reliable over time intervals as long as $2 \mathrm{~min}$, the longest delays tested (Herman \& Gordon, 1974), sensitive to proactive and retroactive interference (although these types of interference did not cause major decrements in performance; Herman, 1975), and capable of maintaining as many as four or five discrete auditory items in parallel (Thompson \& Herman, 1977). Functional similarities of dolphin auditory short-term memory to characteristics of primate visual short-term memory were noted in these studies and were suggestive of a convergence of information-processing characteristics across these large-brained, but evolutionarily divergent, social mammals (see Herman, 1980, for further discussion of convergent information processing

This research was supported by Grant BNS77-16822 and earlier grants from the National Science Foundation to L. M. Herman and is contribution No. 622 from the Hawaii Institute of Marine Biology. Portions of this paper were presented before the Western Psychological Association, Sacramento, California, April 1975. We thank Michael P. Yunker for his assistance in conducting these studies and James Wolz for helpful comments on this paper. Reprint requests should be sent to Louis M. Herman, Department of Psychology, University of Hawaii, 2430 Campus Road, Honolulu, Hawaii 96822. traits of the auditorily specialized dolphin and the visually specialized primate).

In this paper, we continue the description of the auditory memory of the bottlenosed dolphin through study and comparison of performance on identity, symbolic, and probe ("Yes-No") forms of the DMS task. These DMS tasks vary in the number or nature of test stimuli available as postdelay retrieval cues and hence may have different effects on representational (encoding) mechanisms, retrieval processes, or the decisions made at the time of the memory test. Additionally, the DMS data obtained from these tasks allowed for ready comparisons with DMS data already available for monkeys.

For all three DMS procedures of the current study, either of two highly familiar sounds, sound A or sound B, was heard as the sample sound at each discrete DMS trial. These sounds were familiar to the dolphin through their use in a prior study (Thompson \& Herman, 1981) as well as through the large number of DMS training and testing trials given in the current study. For identity matching, after the prescribed delay interval, both familiar sounds were heard (sequentially in either order) as test sounds ( $a$ and $b$ ) and constituted a two-alternative forced-choice delayed recognition test. A response to the alternative matching the prior sample (i.e., $a$ iff $A$ or $b$ iff $B$ ) was reinforced. In symbolic matching, sounds $c$ and $d$ ap- 
peared at the postdelay memory test. Through prior training, sound $\mathrm{c}$ was associated with $\mathrm{A}$, as a symbol for it (cf. D'Amato, 1973), and d was associated with $B$. Hence, a response to $c$ was required if sound $A$ was the sample and a response to $d$ if $B$ was the sample. Finally, in probe matching, a single alternative (a or b) was given at the postdelay test. The single probe might be the same as or different from the sample, so that both identity and nonidentity need be recognized to maximize performance over successive probe memory tests.

Identity matching of two familiar sample sounds was used previously by Herman (1975) and in a visual form in the procedure most commonly used in monkey (see reviews in D'Amato, 1973; Jarrard \& Moise, 1971) and pigeon (see review in Roberts \& Grant, 1976) DMS studies. The DMS procedure of Herman and Gordon (1974) also used identity matching, but the sample sounds at each DMS trial were selected from an open set so that a novel sample sound was given at each DMS trial.

A probe DMS procedure was used by Thompson and Herman (1977) subsequent to this study. Multiple samples, in list form, were given at most DMS trials and were followed by a single probe which either was or was not a member of the previous list. The dolphin was required to classify the probe as "old" (from the list) or "new" (not from the list). The samples for a list were selected quasi-randomly from a pool of 600 sounds such that there was no chance that a sound from a given list repeated a sound from the immediately preceding list. In most cases, repetitions did not occur until the entire pool had been used.

Identity, symbolic, and probe DMS performance have been contrasted previously in visual tests with monkeys (D'Amato \& Worsham, 1974) and provide a comparative framework against which to assess our current findings. D'Amato and Worsham's results indicated that, with sufficient training on each DMS condition, the monkeys attained roughly equivalent levels of performance under the three conditions. Delays generally reached 50 to $100 \mathrm{sec}$ before performance approached chance levels, although one monkey eventually achieved a performance level of $90 \%$ or better correct responses at delays of $120 \mathrm{sec}$. No other species appears to have been tested in these three different DMS paradigms within a single study, although Maki, Moe, and Bierley (1977) reported on delayed identity matching by pigeons of red and green samples and also on delayed symbolic "matching" of red and green test colors to two different fixedratio response "samples" or to food or no-food "samples." Roitblat (1980) also studied identity and symbolic DMS in pigeons, while Nelson and Wasserman (1978) reported on a variant of the probe DMS procedure. In these cited pigeon DMS studies, and in others (see Roberts \& Grant, 1976), the maximum delay attained by the pigeons was typically less than
$10 \mathrm{sec}$, which is well below the limits reported for monkeys or dolphins. However, whether or not memory processes in the pigeon differ in kind from those of the large-brained mammals is still a matter of contention and requires further study (cf. Herman, 1980; Maki et al., 1977; Roberts \& Grant, 1976).

\section{METHOD}

\section{Subjects and Apparatus}

An adult female bottlenosed dolphin named Keakiko (Kea), approximately 9 to 11 years of age at the time of testing, was the subject throughout. She was a test-sophisticated animal, the subject of many earlier studies of hearing capabilities (Herman \& Arbeit, 1972; Thompson \& Herman, 1975; Yunker \& Herman, 1974) and of auditory learning and memory (Herman, 1975; Herman \& Arbeit, 1973; Herman \& Gordon, 1974; Thompson \& Herman, 1981). She was tested in her outdoor home tank, a circular concrete seawater enclosure of $15.2-\mathrm{m}$ diameter with water depth of $1.5 \mathrm{~m}$. Figure 1 is a schematic of the testing apparatus, which was located in one quadrant of the tank. The two pairs of ropes were suspended vertically from beams projecting over the tank and defined an underwater channel leading to the underwater "start" paddle. Kea was required to maintain the anterior portion of her body in the acoustically favorable "listening" area after pressing the start paddle so that she could easily hear the sounds projected from any of the three Chesapeake J9 underwater speakers. The vertically suspended response paddles adjacent to the two peripheral speakers were used by Kea to indicate her postdelay response, as described later.

Two unique sounds served as sample stimuli throughout the experiment. Sound A was a $4-\mathrm{kHz}$ pure tone amplitude-modulated at a rate of $3 \mathrm{~Hz}$ to within $\pm 50 \%$ of its center amplitude. Sound B was an unmodulated $25-\mathrm{kHz}$ pure tone. These same two sounds appeared as postdelay alternative choices ("test" stimuli) in identity DMS, while one or the other appeared in the postdelay tests in probe DMS. Sounds $c$ and $d$ were used as test stimuli for symbolic DMS, and were, respectively, a $15-\mathrm{kHz}$ pure tone frequencymodulated at a $2-\mathrm{Hz}$ rate to within $\pm 10 \%$ of its center frequency and a $7-\mathrm{kHz}$ pure tone frequency-modulated at a $21-\mathrm{Hz}$ rate, again to within $\pm 10 \%$ of its center frequency. A sample sound always

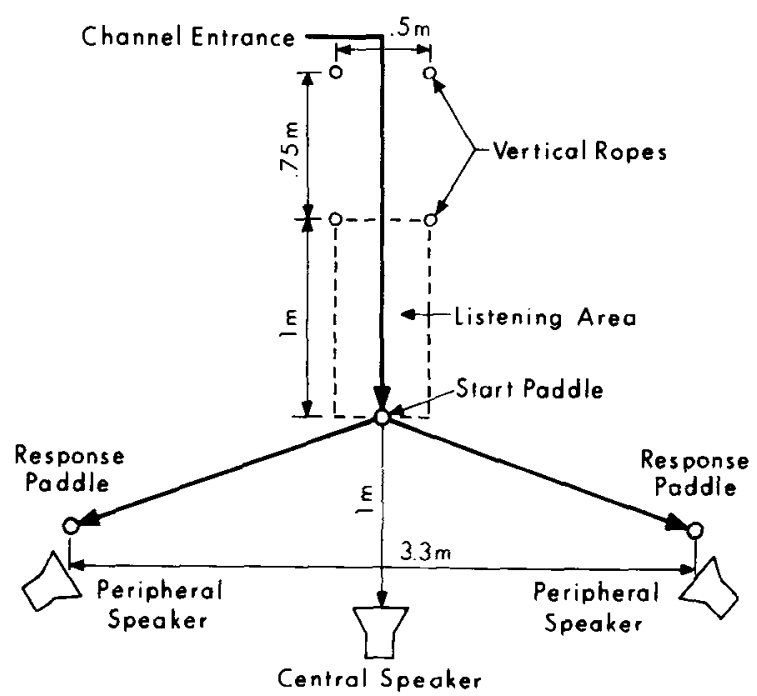

Figure 1. General schematic of the delayed matching-to-sample testing apparatus (not to scale). The speakers, paddles, and terminal ends of the suspended ropes were all anderwater. Thick lines show path of dolphin. 
appeared at the center speaker (see Figure 1) and the test sound(s) at one or each of the two peripheral speakers.

Additionally, three control sounds of complex waveform and of different primary frequencies appeared at the center speaker: $\mathbf{A}$ "call sound" ( $221 \mathrm{~Hz}$ ) controlled Kea's entrance into the listening area at the start of each DMS trial; an "exit sound" (334 Hz) controlled her departure from the listening area to press one of the two response paddles at the postdelay matching test; and a conditionedreinforcer sound $(522 \mathrm{~Hz})$ was used immediately after a press of the correct paddle.

All sample and test sounds were generated by two Wavetek 154 programmable waveform generators digitally controlled by a minicomputer. The control sounds were produced by dedicated generators that had relay outputs sequenced by the computer. The computer also sequenced the discrete trials, timed all sound stimuli and the interstimulus and intertrial intervals, and assigned the various sounds to the proper speakers.

The mean in-water signal levels for sounds $A, B, c$, and $d$ were approximately $35,48,44$, and $38 \mathrm{~dB}$ (re: $1 \mu$ bar), respectively, measured $1 \mathrm{~m}$ from the speaker. All of these signal levels were well above the absolute frequency thresholds reported for Tursiops truncatus (Johnson, 1967).

\section{Procedure}

The same discrete trial procedure was used for all three DMS tasks. Kea's attention to the sample sound, her waiting behavior during the delay period, and other aspects of the DMS procedure were controlled by a chain of responses largely developed in our prior DMS studies (Herman, 1975; Herman \& Gordon, 1974). As in the past, the chain was performed with high reliability.

The chain began with projection of the limited-hold (10-sec) call sound from the center speaker (Figure 1). In response, Kea entered the listening area through the channel of suspended ropes and pressed the start paddle, turning the call sound off. If she failed to press the start paddle within the 10-sec limit, the call sound turned off automatically and a "time-out" of 15 or $30 \mathrm{sec}$ began, corresponding in length to the duration of the intertrial interval (ITI) in that testing session. After the time-out, the call tone began again.

Kea almost always pressed the start paddle within the 10 -sec time limit, except for the infrequent occasions when she selfterminated a session (see later). After the start paddle was pressed successfully, a 4-sec pause began and at its end the sample sound, randomly either sound $A$ or sound $B$, was projected from the center speaker for $4 \mathrm{sec}$. Either sound A or sound B appeared as the sample in a random order that was predetermined, with the construint that no one sample occur for more than four trials in a row. When the sample sound ended, the predetermined delay interval began. It ranged in duration from 1 to $240 \mathrm{sec}$, and at its termination the test sound(s) was (were) projected from the peripheral speakers.

For identity and symbolic matching, there were two test sounds. They occurred sequentially for $2.5 \mathrm{sec}$ each and were separated by a .5 -sec silent interval. One sound appeared at the left-hand speaker and the other at the right (Figure 1). Whether the correct test sound was first or last in the sequence and whether it appeared at the left or right speaker were balanced over trials, using a quasi-random schedule. The exit sound began $.5 \mathrm{sec}$ after the second test sound ended; Kea then left the listening area and pressed a response paddle. A press of the paddle adjacent to the peripheral speaker that had projected the correct test sound (the sound symbolically or identically matching the prior sample) turned off the exit sound and produced $2.0 \mathrm{sec}$ of playback of the matching test sound from the adjacent speaker. This was followed immediately by the .5 -sec conditioned reinforcer sound, after which a whole silver smelt, Osmerus sp., weighing approximately $35 \mathrm{~g}$, was thrown into the tank. Pressing the incorrect response paddle also turned off the exit sound but produced playback of the nonmatching test sound. Playback had previously been shown to be necessary for control of responding to sequentially presented sounds (Herman
\& Arbeit, 1973). The ITI, either 15 or $30 \mathrm{sec}$ in counterbalanced order across successive testing sessions, began $.5 \mathrm{sec}$ after termination of playback for either correct or incorrect responses.

For probe matching, only a single test sound (the probe) was heard. It played for $2.5 \mathrm{sec}$ and on half the occasions appeared at the left-hand speaker and on the remaining half at the right, in a quasi-random sequence over trials. For a correct matching response (probe same as sample), Kea responded to the paddle adjacent to the speaker that projected the probe, exactly as in identity DMS. The standard reward sequence of playback of the selected sound, the conditioned reinforcer sound, and a thrown fish followed. For a correct nonmatching response (probe different from sample), Kea responded on the paddle adjacent to the "silent" speaker, the one that projected no sound. In this case, the matching sound was played back, just as if it had appeared at the silent speaker, followed by the rest of the standard reward sequence. Incorrect responses-responding to the silent speaker given a matching probe or to the active speaker given a nonmatching probe-were not followed by playback. The deletion of playback after incorrect responses was in keeping with the deletion of the sequential test sound condition used with identity and symbolic DMS. The ITI conditions were the same as the previous ones, except that the ITI began $2.5 \mathrm{sec}$ after an incorrect paddle press (to compensate for the deleted playback time).

The three DMS conditions were tested in the order of symbolic, identity, and probe matching, with all training and testing for an earlier condition completed before training for the subsequent condition began. Within each condition, multiple replications were given, each replication providing an estimate of the length of delay that could be achieved by the dolphin. The entire study encompassed 10.5 months. Because of this extended duration, it was not possible to complete a counterbalanced design to control for testing order. However, analyses of replicate effects allowed for detection of any effect of successive tests, within or across conditions.

Training for symbolic matching was by far the most prolonged of the three DMS tasks. Kea experienced considerable initial difficulty in stably maintaining the symbolic relationship. Whether this was because of negative transfer from her prior experience with identity matching (Herman, 1975; Herman \& Gordon, 1974) or because of the inherent difficulty of a symbolic task cannot be determined from our data. However, D'Amato and Worsham (1974) reported that symbolic DMS was a much more difficult task for monkeys to learn than was identity DMS; however, once learned, the monkeys performed as well on symbolic DMS as they did on identity DMS.

To train reliable symbolic matching, more than 7,000 trials were required over a 7 -week period. Training began with $1-\mathrm{sec}$ delays and eventually progressed to considerably longer delays. In summary, the training steps were:

(1) The establishment of reliable and reversible two-choice discriminations between sounds $c$ and $d$ without any sample sound present-This was accomplished with little error through "fading" techniques in which the negative sound $\left(\mathrm{S}_{-}\right)$was initially much shorter in duration than the positive sound $(S+)$. Over successive trials of correct responding within reversals, the $S$ - duration was gradually increased to that of $S+(2.5 \mathrm{sec})$. Each of the first 10 reversals began with the $S-$ at a .5 -sec duration, but over successive reversals the number of trials taken to complete the fading schedule was reduced from 12 trials to 1 trial.

(2) The gradual introduction of the sample sounds A and BReversal training was continued as in (1), but now the sample sounds (A or B) cued each reversal. The sample duration was short $(.5 \mathrm{sec})$ for the first two reversals but, over successive trials of correct responding, its duration was gradually increased to the final value of $4 \mathrm{sec}$. A series of multitrial reversals was then given with $A$ and $B$ at full duration throughout. During these latter reversals, the $\mathrm{S}$ - was also maintained at its full $2.5 \mathrm{sec}$ duration for all trials, including Trial 1.

(3) The random presentation of sound A or sound B as sampleA gradual changeover was made from long blocks of trials per 
reversal to shorter blocks of trials and, finally, to random presentations of the two sample sounds over successive trials. During the final 11 sessions of 96 trials each, with sound A or sound B occurring as sample in random order over trials, Kea's performance per session remained between $87.5 \%$ and $96.0 \%$ correct.

(4) Familiarization with longer delays-The final step incremented the delay limit gradually beyond the 1-sec values used in (2) and (3). Increments were at first in units of only .1 or .2 sec, but later they were in units of up to $2.0 \mathrm{sec}$. Increments were made after correct responses on at least 11 of 12 consecutive trials, while three or more errors in a block of 12 trials resulted in a decrease in the delay-value duration by one step. A total of 68 96-trial sessions, usually 2 sessions per day, were given in this manner. The maximum delay interval reached was $74 \mathrm{sec}$ before Kea's increasing error rate resulted in her refusal to answer the call tone, a behavioral tendency that was to reappear in later testing as well. At the next session, formal symbolic DMS testing began with delays of 1 and $2 \mathrm{sec}$. A new delay-incrementing procedure was used as described in the later section on testing.

Training for identity matching was simple relative to the requirements for training symbolic matching. Only 576 trials were given over a 3-day period in six 96-trial training sessions. The $\mathrm{S}-$ was cued by its short duration $(.5 \mathrm{sec})$ on Trial 1 during Sessions 1 through 3, but its duration was eliminated as a cue in the remaining sessions. Sample sounds A and B were at their full 4-sec duration from the beginning, and the delay interval was maintained at $1 \mathrm{sec}$ throughout. Successive reversals, each of 4 to 12 trials, were given for the first five sessions. In the sixth, and final, training session, blocked trials were eliminated and sound A or sound B appeared as the sample in a quasi-random sequence over the 96 trials. The results were that Kea made 16 matching errors in Session 1, 11 in Session 2, and then 1, 0, 1, and 2 errors, respectively, in each of the remaining four 96-trial sessions. There was no attempt to give experience with delays greater than $1 \mathrm{sec}$ prior to the start of formal identity DMS testing, as Kea was already familiar with longer delays through her symbolic DMS testing.

Transfer to probe DMS from the preceding identity DMS condition was, surprisingly, immediate. Kea made no errors during a single 48-trial training session in which the second test sound in the sequence was gradually deleted by decreasing its duration in $.2-\mathrm{sec}$ steps after every six consecutive correct responses. During this session, the sample sounds were presented in random sequence across trials at their full 4-sec duration. It had been observed during the prior identity matching tests that Kea often oriented towards the correct response paddle before the second test sound began, indicating that she was already able to respond correctly on the basis of a single matching or nonmatching probe sound. Actually deleting the second test sound thus had little influence on her performance.

The testing procedure for all three DMS conditions consisted of incrementing the delay interval from initial 1 - and 2 -sec values according to a predetermined schedule (Table 1) until a stopping criterion-based either on errors $(50 \%$ correct responses or less in a block of $\mathbf{2 4}$ trials, six consecutive errors, or biased responding to only one test sound) or on self-termination by Keawas met. After a stop, the delay schedule was replicated from the beginning to obtain another estimate of delay limit. Using this procedure, the symbolic DMS condition was replicated twice (two iterations of the delay schedule in Table 1), the identity condition five times, and the probe four times. The additional replications under the last two conditions took into account their attenuated training time as compared with symbolic matching. In fact, as described in the Results section, performance on later replications differed unsystematically from that on earlier replications.

Table 1 is most easily interpreted by the example for 1 - and 2 -sec delays. This pair of delays was used for 96 successive DMS trials that constituted a testing session. The two delays appeared in a quasi-random balanced sequence across the 96 trials. If neither stopping criterion was met in the 96 trials, the longer $(2-\mathrm{sec})$ delay of the pair was used at the next session together with an incremented
Table 1

Pairs of Delays (in Seconds) Used at Successive Testing Sesslons and the Number of Trials per Session

\begin{tabular}{ccccccccc}
$\begin{array}{c}\text { Delay } \\
\text { Lengths }\end{array}$ & Trials & $\begin{array}{c}\text { Delay } \\
\text { Lengths }\end{array}$ & \multicolumn{3}{c}{$\begin{array}{c}\text { Trials } \\
\text { Lelay }\end{array}$} & $\begin{array}{c}\text { Lengths } \\
\text { Trials }\end{array}$ & $\begin{array}{c}\text { Delay } \\
\text { Lengths }\end{array}$ & Trials \\
\hline $1-2$ & 96 & $10-12$ & 96 & $34-40$ & 72 & $90-100$ & 48 \\
$2-3$ & 96 & $12-14$ & 96 & $40-46$ & 72 & $100-110$ & 48 \\
$3-4$ & 96 & $14-16$ & 96 & $46-52$ & 72 & $110-120$ & 36 \\
$4-5$ & 96 & $16-18$ & 96 & $52-58$ & 48 & & \\
$5-6$ & 96 & & & & & & \\
$6-7$ & 96 & $18-22$ & 96 & $58-66$ & 48 & $120-180$ & 36 \\
$7-8$ & 96 & $22-26$ & 96 & $66-74$ & 48 & $180-240$ & 36 \\
$8-9$ & 96 & $26-30$ & 96 & $74-82$ & 48 & & \\
$9-10$ & 96 & $30-34$ & 72 & $82-90$ & 48 & & \\
\hline
\end{tabular}

Note-The above schedule is for a single replication beginning with delay lengths of $1.2 \mathrm{sec}$. The interval between pairs of delays increases at each column and also across breaks within columns.

(3-sec) delay. Additional sessions were carried out in a comparable manner, with the number of trials per session as given in Table 1 . As the pair of delays increased in duration, the number of trials per session was decreased in order that the sessions not be inordinately long. There were generally two sessions per day separated by a 1- to 2-h interval. A 15 - to 20 -min break was given halfway through each session.

\section{RESULTS}

\section{Delay Limitations and Delay Effects}

Table 2 summarizes performance on the next-tolast and last delay block reached during each replication for each of the three DMS conditions. There was no systematic trend across replications in any condition. Apparently, the extensive DMS training given Kea in this and earlier studies (e.g., Herman, 1975) made the further practice obtained during DMS test-

Table 2

The Delay Pairs Reached by Ken on the Next-to-Last and Last Sessions of Each Replication

\begin{tabular}{|c|c|c|c|c|c|c|c|}
\hline \multirow{2}{*}{$\begin{array}{l}\text { Repli- } \\
\text { cation }\end{array}$} & \multicolumn{3}{|c|}{ Next-to-Last Session } & \multicolumn{3}{|c|}{ Last Session } & \multirow{2}{*}{$\begin{array}{l}\text { Stop } \\
\text { Rule }\end{array}$} \\
\hline & Pair & Trials & $\% \mathrm{C}$ & Pair & Trials & $\% \mathrm{C}$ & \\
\hline \multicolumn{8}{|c|}{ Symbolic DMS } \\
\hline 1 & $120-180$ & 35 & 80.0 & $180-240$ & 10 & 90.0 & $S$ \\
\hline 2 & $110-120$ & 36 & 91.7 & $120-180$ & 8 & 62.5 & $S$ \\
\hline \multicolumn{8}{|c|}{ Identity DMS } \\
\hline 1 & $110-120$ & 36 & 94.4 & $120-180$ & 6 & 66.7 & $S$ \\
\hline 2 & $26-30$ & 96 & 100.0 & $30-34$ & 36 & 66.7 & $E$ \\
\hline 3 & $120-180$ & 36 & 100.0 & $180-240$ & 6 & 66.7 & $S$ \\
\hline 4 & $58-66$ & 38 & 81.6 & $66-74$ & 4 & 25.0 & $\mathbf{S}$ \\
\hline 5 & $110-120$ & 36 & 91.7 & $120-180$ & 14 & 100.0 & $S$ \\
\hline
\end{tabular}

\begin{tabular}{rrrrrrrr}
\multicolumn{7}{c}{ Probe DMS } \\
1 & $66-74$ & 24 & 87.5 & $74-82$ & 36 & 88.9 & $\mathrm{~S}$ \\
2 & $30-34$ & 72 & 98.6 & $34-40$ & 44 & 72.7 & $\mathrm{~S}$ \\
3 & $58-66$ & 48 & 93.8 & $66-74$ & 17 & 58.8 & $\mathrm{E}$ \\
4 & $82-90$ & 48 & 91.7 & $90-100$ & 13 & 53.8 & $\mathrm{~S}$ \\
\hline
\end{tabular}

Note-\%C=percent correct; $E=$ error criterion met; $S=$ self termination by Kea. 
ing of little importance for performance. This was unlike the results in our very first DMS study with Kea (Herman \& Gordon, 1974) in which there was marked improvement in the delay limits reached and in the reliability of performance during the course of DMS testing (cf. D'Amato \& Cox, 1976). The lack of an ordered replication effect within or across DMS conditions in the current studies makes it unlikely that the sequence in which the three conditions were given affected the obtained results. This point is taken up again later.

In only 2 of the 11 replications over all conditions was a stopping criterion met for reasons other than self-termination. Table 2 shows that some exceptionally long delay intervals-e.g., 180 and $240 \mathrm{sec}-$ were reached by Kea for at least a few trials before self-termination. The principal performance constraint was therefore not necessarily a memory limitation but, rather, Kea's refusal to continue with the testing after errors at long delays, and occasionally after relatively shorter delays.

Figure 2, a semilogarithmic plot, compares performance across the three DMS conditions. Shown is the percentage of correct responses at each delay interval given. To obtain the most reliable estimate of performance at each delay value, the data for all replications were pooled within each DMS condition. Addi-
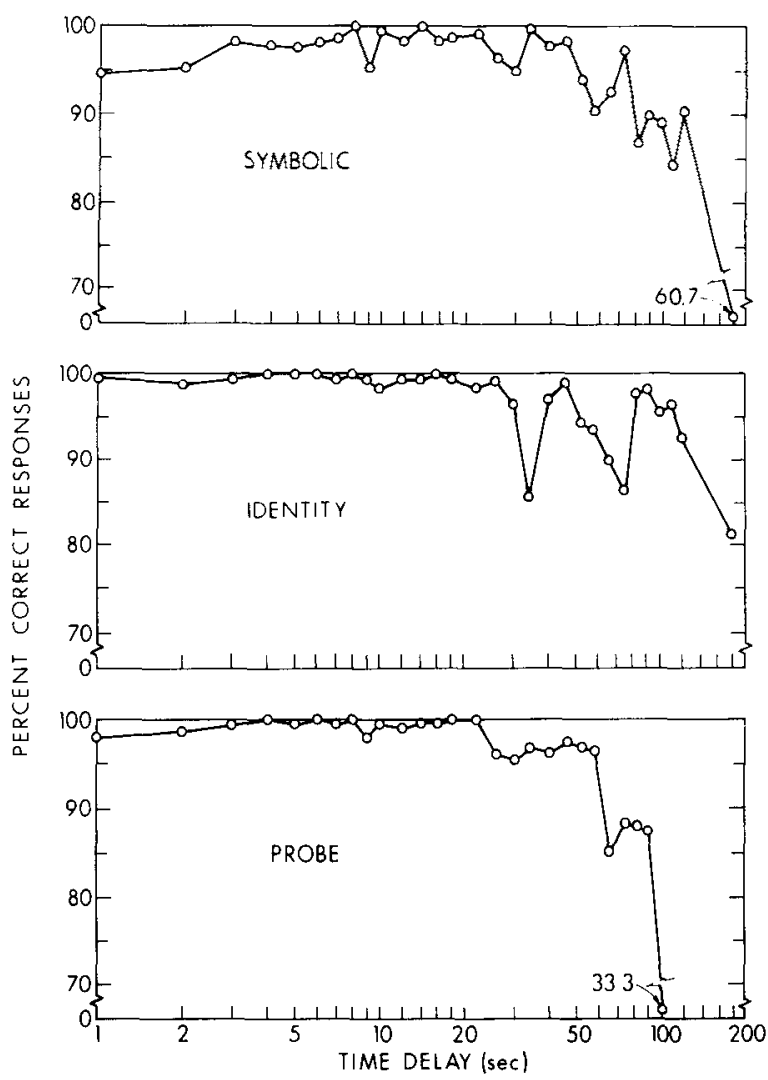

Figure 2. The percentage of correct responses as a function of delay interval for each of three DMS procedures. tionally, data for the 15 - and 30 -sec ITI values were combined, since the ITI variable did not produce significant effects (see later).

The number of trials per data point in Figure 2 was generally very large, except at the longest delays. For delays of $58 \mathrm{sec}$ or less, there were at least 96 trials and as many as 480 in some cases. There were at least 44 trials for all delays up to $90 \mathrm{sec}$. The fewest number of trials represented in Figure 2 was 6 for the 100 sec delay value in probe DMS and 28 and 26 , respectively, for the $180-\mathrm{sec}$ value in symbolic and identity DMS. Not shown in Figure 2 are the results for 5 trials at $240 \mathrm{sec}$ in symbolic DMS $(80 \%$ correct) and three trials at $240 \mathrm{sec}$ in identity DMS (66.7\% correct).

From Figure 2, it is apparent that the general performance characteristics across the three DMS conditions were very similar. The percentage of correct responses tended to remain between $95 \%$ and $100 \%$ through to delays of $46 \mathrm{sec}$, decreased irregularly thereafter, but never fell below $80 \%$ through to at least a 90-sec delay. In keeping with the fact that termination of 9 of the 11 replications was not due to an error-stopping criterion being met, Kea's performance on all but two of the delays longer than $90 \mathrm{sec}$ was also above $80 \%$ correct.

\section{Effects of ITI, Sample Sounds, and Test Sounds}

Table 3 summarizes the effects of the indicated variables on DMS performance. Within each variable and DMS condition, the data were blocked by groups of delay values to reveal any consistency of trends, since error rates at any single delay were so small. However, all tests of significance in Table 3 were made on the effects over the combined delays (on the row labeled "ALL")."

Table 3 shows that the ITI variable did not yield significant effects in any DMS condition. Unlike prior data on identity matching by Kea (Herman, 1975), no significant ITI effect was found. However, ITIs in the 1975 study ranged from 7.5 to $60 \mathrm{sec}$ in duration. The lack of an ITI effect in the current study might have reflected the restriction on range of ITI or the absence of a very short ITI, which is potentially the most interfering (e.g., see Jarrard \& Moise, 1971, Figure 9, for ITI effects on visual DMS by monkeys, and Nelson and Wasserman, 1978, for ITI effects on pigeons).

Additional comparisons in Table 3 include response errors to the two different sample sounds and, in the case of symbolic and identity DMS, the effects of the order of presentation of the two test sounds. For probe DMS, in which only one test sound (the probe) was given per trial, the analysis focused on whether performance differed when probes matched the samples (matching case) from when they did not (nonmatching case). 
Table 3

Percentage of Errors in Each DMS Condition as a Function of Intertrial Interval (ITI), Sample Sound Used (A or B), Order of Test Sound (First or Second), and Whether Probe Matched or Did Not Match the Sample

\begin{tabular}{|c|c|c|c|c|c|c|c|c|}
\hline \multirow[b]{2}{*}{ Delay } & \multicolumn{2}{|c|}{ ITI } & \multicolumn{2}{|c|}{ Sample } & \multicolumn{2}{|c|}{ Order } & \multicolumn{2}{|c|}{$\begin{array}{l}\text { Probe } \\
\text { Sound }\end{array}$} \\
\hline & 15 & 30 & A & B & 1 & 2 & M & $\mathrm{NM}$ \\
\hline
\end{tabular}

Symbolic DMS

$1-5$
$6-10$
$12-18$
$22-34$
$40-58$
$66-120$
All

$\begin{array}{rrr}3.3 & 3.0 & 4.2 \\ .8 & 1.8 & 1.6 \\ 1.2 & 1.0 & 1.2 \\ 2.4 & 2.6 & 2.4 \\ 4.8 & 3.2 & 4.0 \\ 9.9 & 9.9 & 7.8 \\ 3.2 & 3.1 & 3.1\end{array}$

$\begin{array}{lll}2.1 & .3 & 6.0\end{array}$

$\begin{array}{lll}1.0 & 0 & 2.6\end{array}$

$\begin{array}{lll}1.0 & .2 & 2.0\end{array}$

$\begin{array}{lll}2.6 & 1.1 & 3.9\end{array}$

$\begin{array}{lll}4.0 & 4.4 & 3.6\end{array}$

$\begin{array}{lll}11.9 & 8.6 & 11.1\end{array}$

$3.2 \quad 1.9+4.4$

Identity DMS

$\begin{array}{rrrcrrr}1-5 & .8 & .3 & 0 & 1.1 & .7 & .4 \\ 6-10 & .6 & 1.3 & .4 & 1.4 & 1.0 & .9 \\ 12-18 & .5 & .2 & 0 & .7 & .4 & .4 \\ 22-34 & 2.3 & 3.8 & 2.6 & 3.5 & 3.3 & 2.8 \\ 40-58 & 5.1 & 4.0 & 2.7 & 6.5 & 3.8 & 5.3 \\ 66-120 & 3.6 & 4.2 & 4.4 & 3.4 & 3.0 & 4.8 \\ \text { All } & 1.9 & 2.1 & 1.5+ & 2.5 & 1.9 & 2.2\end{array}$

Probe DMS

\begin{tabular}{rrrrrcr}
$1-5$ & .8 & 1.2 & 1.0 & 1.0 & 0 & 2.0 \\
$6-10$ & .5 & .7 & .3 & .8 & .9 & .3 \\
$12-18$ & .3 & .4 & .2 & .6 & 0 & .8 \\
$22-34$ & 2.0 & 3.9 & 2.8 & 3.1 & .3 & 5.7 \\
$40-58$ & 2.7 & 3.5 & 2.3 & 3.8 & .7 & 5.4 \\
$66-100$ & 10.3 & 22.1 & 10.2 & 18.9 & 5.3 & 24.0 \\
All & 1.8 & 2.5 & $1.7 *$ & 2.6 & $.6 \quad+\quad 3.7$ \\
\hline
\end{tabular}

Note-Delay and ITI are given in seconds. $M=$ match; $N M=$ nonmatch. Paired values that are underlined differ significantly. ${ }^{*} \chi^{2}(1)=5.49, p<.01 . \quad t_{\chi}{ }^{2}(1) \geqslant 11.18, p<.001$.

The results of the analyses were that significantly more errors occurred with sound $B$ as the sample than with sound $A$ for both identity and probe DMS but not for symbolic DMS. For symbolic DMS, significantly more errors were made when the correct test sound was last in the sequence than when it was first. A slight, but nonsignificant, trend in this direction was observed in the identity DMS condition. Sequence effects of test sounds are of course not applicable to probe DMS. For probe DMS, errors were significantly greater when the probe was different from the sample ("false alarm" error) than when it was the same as the sample ("missed detection" error), with the relative effects greatest at the longer delays. Overall, the results of Table 3 indicate that response tendencies or biases may shift to some degree from one DMS procedure to the next, affecting some of the fine-grain interpretation of memory-dependent processes, but that the different procedures have little influence on the level of performance reached.

\section{Interference Effects from Prior Trial}

Effects of changing the sample sound. With small, highly familiar sample sets, the prior-trial sample may influence the choice of test sound on the current trial (Herman, 1975; Worsham, 1975). Figure 3 compares error rates associated with sample values that were the same as those of the prior trial (i.e., A-A or $B-B)$ with error rates for sample values that were different from those of the prior trial (i.e., A-B or B-A). The interference from changing the prior-trial sample described by Herman (1975) appears consistently in Figure 3 for both the symbolic and identity DMS conditions. For each delay block, changing the sample sound across successive trials was associated with a greater error rate (shaded bars) than was not changing it (unshaded bars). For symbolic DMS, the overall ("ALL") error rate for changed samples was $3.88 \%$, and for unchanged samples, it was $2.0 \%\left[\chi^{2}(1)=\right.$

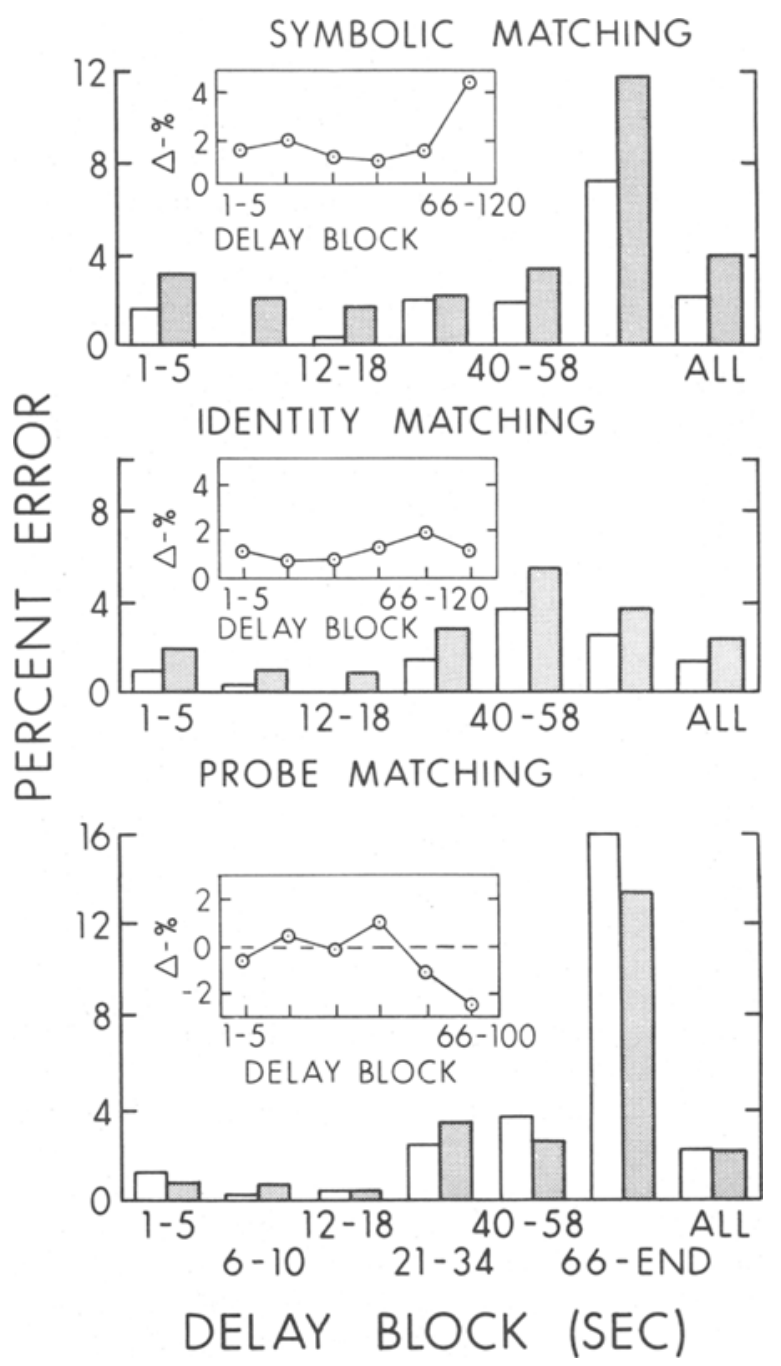

Figure 3. The percentage of error when sample sounds were changed (shaded bars) across successive paired trials and when they were not (unshaded bars), for each of the three DMS procedures. The inset graphs show the differences in error percentages ( $\%$ of shaded bars minus $\%$ of unshaded bars) within each delay block. Note that the final delay block for probe matching is 66100 sec, as compared with 66-120 sec for symbolic and identity matching. 
$10.2, \mathrm{p}<.001]$. For identity DMS, the corresponding percentages were 2.33 and $1.22\left[\chi^{2}(1)=12.5\right.$, $\mathrm{p}<.001]$. The insets in the symbolic and identity matching graphs, which give the differences in the error percentages within delay blocks ( $\%$ error for changed samples minus \% error for unchanged samples), do not indicate a systematic increase in relative error as the delays lengthen. The sudden increase in relative error at the longest delay block in symbolic DMS is an exception to the otherwise flat function seen in the prior portion of that graph or in the identity DMS graph. Generally, strict temporaldiscrimination hypotheses of delayed matching (D'Amato, 1973) would predict a monotonically increasing relative error rate with increasingly long delays, at least within the limits of delay used here.

The interference from changing the prior-trial sample, so evident in identity and symbolic DMS, does not appear in the probe DMS data of Figure 3. Instead, for four of the six delay blocks in probe DMS, and for all blocks combined, the percentage of error was somewhat less when samples were changed across successive paired trials than when they were retained. However, the overall effect was not significant $\left[\chi^{2}(1)=.04, \mathrm{p}>.05\right]$.

These findings suggest that some memory processes or strategies underlying probe DMS performance may diverge from those in symbolic or identity DMS. Since probe DMS differs from the other two DMS procedures in providing only a single test sound and therefore in requiring both identity and nonidentity decisions, a separate analysis for an effect of the prior-trial sample was made for matching and for nonmatching trials. For the total of 2,990 matching probe trials given to Kea, the error percentage associated with a change of sample sound was .60 and with no change it was $.26\left(\chi^{2}=1.82, p>.05\right)$. For the 3,038 nonmatching trials given, the corresponding error percentages were 3.40 for changed samples and 4.12 for unchanged samples $\left(\chi^{2}=.92\right.$, $\mathrm{p}>.05)$. Thus, although the type of DMS trialmatching or nonmatching-affected Kea's overall error rate differentially, as noted earlier, neither type revealed a significant effect of the prior-trial sample on current trial performance or even a consistent trend in that direction.

Effects of testing order of DMS conditions on interference from the prior trial sample. As noted earlier, the extremely lengthy testing procedures for each condition precluded carrying out a counterbalanced design to control for testing order. We showed previously, in Table 2, that there was no practice effect on error rate within replications of a DMS condition. Here, we examine for replicate influences on the effect of the prior-trial sample. The key question is whether the interfering effect of changing the prior-trial sample gradually dissipated across successive replications and across successive DMS conditions. If interference did decrease with practice or with some other time-dependent variable, then the absence of the effect in probe DMS would be theoretically less interesting. Table 4 shows, however, that interference from the prior-trial sample was not influenced by replications or by testing order. In Table 4, the error rates are shown for changed and for retained sample sounds for each replication within each DMS condition. The important column is labeled "relative error" and shows the normalized difference between the error percentages for changed and retained samples. The larger the positive magnitude of the relative error, the greater was the interference caused by changing the sample sound from its value on the preceding trial. Negative relative magnitudes indicate that changing the sample sound in fact led to less error than did retaining it. The two replications in the symbolic DMS condition do not allow for any clear statement of trend, since either ordering (a reduction in interference or an increase in interference) could have occurred by chance alone with .5 probability. Results for the five replications in identity DMS show, however, that interference increased across the first four replications (increasingly large positive magnitudes of relative error) and then decreased slightly at the fifth replication.

The probe DMS results are very different from those of symbolic or identity DMS. Negative values appear in three of the four probe replications, but

Table 4

Percentage of Error and Relative Error for Each Replication as a Function of Changing or Retaining the Sample Sound Across Successive Paired Trials

\begin{tabular}{lccc}
\hline $\begin{array}{c}\text { Replication } \\
\text { Number }\end{array}$ & $\begin{array}{c}\text { Sercent Error } \\
\text { Sample } \\
\text { Changed }\end{array}$ & $\begin{array}{c}\text { Sample } \\
\text { Retained }\end{array}$ & $\begin{array}{c}\text { Relative } \\
\text { Error }\end{array}$ \\
\hline & \multicolumn{2}{c}{ Symbolic DMS } & \\
1 & 3.97 & 1.58 & .60 \\
2 & 3.81 & 2.36 & .38 \\
Both & 3.88 & 2.00 & .49 \\
& \multicolumn{2}{c}{ Identity DMS } & \\
1 & 3.49 & 2.11 & .40 \\
2 & 2.51 & 1.31 & .48 \\
3 & 1.81 & .92 & .49 \\
4 & 1.79 & .65 & .63 \\
5 & 1.57 & .73 & .54 \\
All & 2.33 & 1.22 & .48 \\
& \multicolumn{2}{c}{ Probe DMS } & \\
1 & 1.78 & 2.03 & -.14 \\
2 & 6.84 & 5.17 & .24 \\
3 & .71 & 1.05 & -.48 \\
4 & 1.39 & 1.89 & -.36 \\
All & 2.03 & 2.11 & -.04 \\
\hline
\end{tabular}

Note-Relative error is defined as the value of the percent error for "sample changed" minus the corresponding value of the percent error for "sample retained," with the quantity divided by the percent error for "sample changed." See text for interpretation of quan tities obtained. 
without any systematic trend otherwise. These data strongly suggest that the probe condition, and not the order of testing, was the key instrument in producing the noted difference in the effect of the prior-trial sample in probe DMS from that observed in symbolic or identity DMS.

Effects of probe changes on error rates. A final search for prior-trial effects in probe DMS took into account that the probe sound can change across successive trials independently of the sample sound. Table 5 shows comparisons of the four possible combinations of sample sound and probe sound changes across paired trials (two-trial states): (1) a change in neither sample sound nor probe from that of the prior trial (both retained); (2) a change in sample sound only (probe retained); (3) a change in probe only (sample retained); and (4) a change in both (neither retained). Differences in the overall number of paired trials of each type reflect restrictions in run length of the same sample sound in our trial-randomization technique (which was the case for all of our DMS conditions). The differences in the percentage of error across the four types of two-trial states for nonmatching trials (trials on which the probe did not match the sample) were significant $\left[\chi^{2}(3)=8.5, p<\right.$ $.05]$. For matching trials (probe matched the sample), the corresponding differences were not significant $\left[\chi^{2}(3)=2.69\right]$.

The error trends within the nonmatching trials are revealed by the paired signed values in Table 5 . The first of the paired signs indicates whether the sample value of the prior trial was "supportive" of $(t)$ or nonsupportive of $(-)$ the current-trial sample. Support occurs when the sample value was retained across successive paired trials, and nonsupport, when the sample value was changed. The second of the paired signs makes a similar analysis for the probe. A sup- porting probe $(+)$ occurs when the probe value was changed across successive paired trials so that the current-trial sample and the prior-trial probe have the same value. A nonsupporting probe (-) refers to the case in which the probe value was retained across successive paired trials so that the current-trial sample and the prior-trial probe have different values. With these definitions in mind, it is easy to see in Table 5 that error rates were greatest when the probe was nonsupportive, regardless of the support or nonsupport of the sample. Combining the two cases in which the probe was retained yielded an error rate of $4.8 \%$; this compares with an error rate of $2.8 \%$ for the two conditions when the probe was changed. The difference between these two error rates was highly significant $\left[\chi^{2}(1)=8.22, p<.01\right]$.

A parallel analysis for sample effects across successive paired trials yielded $4.12 \%$ error when sample values were supportive (sample values retained) and $3.40 \%$ when they were nonsupportive (sample values changed). The difference in error rate was not significant $\left[\chi^{2}=.92\right]$.

The influence of the probe did not hold for the matching probe trials. Instead, on these trials there was a trend for nonsupporting samples to be associated with greater error rates, regardless of the support or nonsupport of the probe. However, it should be recalled that the overall effects of manipulating prior-trial probes and/or samples during matching trials was nonsignificant and that the overall error rate on these trials was remarkably small. We may conclude from Table 5 that in probe DMS a priortrial effect was present during nonmatching trials, that it produced a relatively large error rate overall, and that the interfering effect was traceable to priortrial probes that were nonsupportive of the currenttrial sample.

Table 5

Synopsis of Error Rates Associated With Changing or Retaining Sample and/or Probe Over Successive Paired Trials (Two-Trial State)

\begin{tabular}{|c|c|c|c|c|c|c|c|c|c|c|}
\hline \multirow[b]{2}{*}{$\begin{array}{c}\text { Two-Trial } \\
\text { State }\end{array}$} & \multicolumn{5}{|c|}{ Nonmatching Probe (Current Trial) } & \multicolumn{5}{|c|}{ Matching Probe (Current Trial) } \\
\hline & $\begin{array}{l}\text { Prior } \\
\text { Trial }\end{array}$ & & $\begin{array}{c}\text { Current } \\
\text { Trial }\end{array}$ & $\begin{array}{l}\text { Number } \\
\text { of Pairs }\end{array}$ & $\begin{array}{c}\text { Percent } \\
\text { Errors }\end{array}$ & $\begin{array}{l}\text { Prior } \\
\text { Trial }\end{array}$ & & $\begin{array}{c}\text { Current } \\
\text { Trial }\end{array}$ & $\begin{array}{l}\text { Number } \\
\text { of Pairs }\end{array}$ & $\begin{array}{c}\text { Percent } \\
\text { Errors }\end{array}$ \\
\hline Both S and P Retained & $\begin{array}{l}A-b \\
B-a\end{array}$ & $(+)(-)$ & $\begin{array}{l}A-b, 0 \\
B-a, 0\end{array}$ & 511 & 5.3 & $\begin{array}{l}A-a \\
B-b\end{array}$ & $(+)(+)$ & $\begin{array}{l}\mathbf{A}-\mathbf{a}, 0 \\
\mathbf{B}-\mathbf{b}, 0\end{array}$ & 425 & .2 \\
\hline Only P Retained & $\begin{array}{l}B-b \\
A-a\end{array}$ & $(-)(-)$ & $\begin{array}{l}A-b, 0 \\
B-a, 0\end{array}$ & 831 & 4.5 & $\begin{array}{l}B-a \\
A-b\end{array}$ & $(-)(+)$ & $\begin{array}{l}\mathbf{A}-\mathbf{a}, 0 \\
\mathbf{B}-\mathbf{b}, 0\end{array}$ & 628 & .8 \\
\hline Only S Retained & $\begin{array}{l}A-a \\
B-b\end{array}$ & $(+)(+)$ & $\begin{array}{l}\text { A-b,0 } \\
\text { B-a }, 0\end{array}$ & 556 & 3.1 & $\begin{array}{l}A-b \\
B-a\end{array}$ & $(+)(-)$ & $\begin{array}{l}A-a, 0 \\
\text { B-b,0 }\end{array}$ & 741 & .3 \\
\hline Neither P nor S Retained & $\begin{array}{l}\text { B-a } \\
A-b\end{array}$ & $(-)(+)$ & $\begin{array}{l}A \cdot b, 0 \\
B-a, 0\end{array}$ & 1140 & 2.6 & $\begin{array}{l}B-b \\
A-a\end{array}$ & $(-)(-)$ & $\begin{array}{l}A-a, 0 \\
B-b, 0\end{array}$ & 1196 & .5 \\
\hline
\end{tabular}

Note-S = sample sound; $P=$ probe sound. $A$ and $B$ refer to the sample value, and lowercase $a$ and $b$ refer to the probe yahe. The correct responses on the current trial are a response to the silent speaker (" 0 ") in the nonmatching case or a response to the speaker projecting the matching probe (" $a$ " or " $b$ ") in the matching case. The first of each pair of signs (t or -1 signifies whether the sample of the prior trial is supportive (+) or nonsupportive (-) of a correct response on the current trial; the second of the two signs makes a comparable analysis for the probe of the prior trial (see text). 


\section{DISCUSSION}

The results indicated that the bottlenosed dolphin tested was capable of updating and faithfully maintaining information about the last sample sound heard over intervals at least as long as 2 to $4 \mathrm{~min}$. The DMS tasks studied here all used the same two familiar sample sounds throughout, and only the relative recency of their occurrence as sample needed to be updated (D'Amato, 1973; Mason \& Wilson, 1974). Their physical attributes were already well learned and presumably registered in permanent storage (cf. Herman \& Gordon, 1974). There was little evidence that the maximum delay values reached approached a true upper limit of Kea's DMS capabilities. As shown in Table 3 , all but 2 of the 11 replications ended when Kea refused to continue with additional DMS trials. Although almost all of the selfterminations were after one or several errors, in one case Kea refused to continue a DMS session after 14 correct trials in a row at delays of 120 and $180 \mathrm{sec}$. Apparently the costs of a long wait at times outweighed the subjective benefits of rewarded patience. Possibly, increased reward magnitude at the longer delays might have extended Kea's patience, and her DMS performance, but we did not test this possibility.

Within the limits of the delays tested and the procedures used, there was little difference in the performance characteristics across the three DMS tasks. Self-terminations tended to occur earlier in probe DMS than in the other two conditions, but, overall, the functional characteristics of the three performance curves in Figure 2 were very similar. Kea was approximately as effective at symbolic matching after delays as she was at identity matching and could categorize both similarity and difference relationships correctly in probe DMS (cf. Thompson \& Herman, 1977). These capabilities reveal considerable flexibility in the encoding, maintenance, and retrieval of auditory information by the bottlenosed dolphin and add to other evidence of the extensive auditory learning and memory skills in this largebrained auditorily specialized social mammal (Herman, 1980). That Kea experienced initial difficulty in forming stable symbolic relationships may indicate some limitation in capabilities for developing associations between purely arbitrary events like the computer synthesized sounds of this study.

In many of its functional attributes, the performance of the dolphin on its auditory DMS tasks was reminiscent of the performance of monkeys tested in analogous visual DMS tasks (cf. D'Amato \& Worsham, 1974). The capuchin monkeys tested by D'Amato and Worsham successfully matched visual sample information over delay intervals comparable to those reported here in identity, symbolic, and probe DMS tasks (the sample set in identity and probe DMS comprised four familiar visual samples, and two of these four were used in symbolic DMS). Furthermore, although there was little difference in final performance levels across the three DMS tasks, like the results shown here, the monkeys had great difficulty in their initial learning of the symbolic relationships. Also, like our current data for identity DMS and the earlier data of Herman (1975), Worsham (1975) reported increased error rates in visual delayed identity DMS by monkeys when samples were changed across successive trials. As noted earlier, the comparable pigeon data for stimulus matching in identity, symbolic, and probe DMS procedures are not available. Studies investigating one or two of these DMS conditions with pigeons generally report a rapid decline in DMS performance to chance levels within delay durations of only $10 \mathrm{sec}$ (e.g., Maki et al., 1977; Nelson \& Wasserman, 1978; Roberts \& Grant, 1976). Unlike the dolphin or monkey, pigeons have learned symbolic DMS as readily as identity DMS (Roitblat, 1980). However, the maximum delays tested were less than $6 \mathrm{sec}$. For dolphin (this study) or monkey (D'Amato \& Worsham, 1974), the instability in performance of symbolic DMS early in training occurred primarily when attempts were made to stretch delays, even a few seconds, from starting values of 0 or $1 \mathrm{sec}$. The available data are not sufficient to judge whether analogous effects may have obtained with pigeons.

Grant and Roberts (1976) noted in their pigeon visual-identity DMS work that changing or not changing the sample across successive trials had almost no differential effect on performance. This is unlike the current or past data for the dolphin or data for the monkey (Worsham, 1975) and provides another point of contrast between characteristics of pigeon short-term memory, as measured by DMS tasks, and dolphin and monkey short-term memory. Herman (1980) and Roberts and Grant (1976) have discussed other points of memory contrast between this avian representative and mammals as represented by the relatively large-brained monkey or dolphin. These memory performance differences between the chosen avian and mammalian representatives might reflect a general avian-mammalian contrast, or a "largebrained" vs. "small-brained" distinction, or they might index the effects of adaptive cognitive specializations of primate and dolphin that are not evident in other mammals or in nonmammalian forms. Herman (1980) stressed that memory characteristics of other representatives of avians and mammals must be mapped out before one can conclude that a general avian-mammalian memory distinction is involved, as was postulated by Roberts and Grant (1976).

D'Amato (1973) and Dewson and Burlingame (1975) have supposed that the symbolic matching task might measure recall memory. Since the samples do not reappear at the postdelay memory test-only the conditionally related alternatives are given-the 
animal must presumably "recall" rather than "recognize" the prior sample in order to make a match. However, this interpretation neglects the advantages offered by coding processes. Coding and coding processes have been discussed extensively in analyses of animal (and human) memory (Blough, 1959; Gaffan, 1977; Honig, 1978; Roitblat, 1980; Thompson \& Herman, 1981). Thus, it seems as reasonable to suppose that the animal (dolphin or monkey) immediately represents the sample in memory by its associated symbol, and maintains it that way over time, as to suppose that such transformations are made only at the moment of the matching test. Roitblat (1980) gave evidence that pigeons maintain a representation of the symbol rather than the sample. If input coding of sample events takes place as postulated here, then recognition processes can govern decisions at the postdelay symbolic DMS test and symbolic DMS becomes functionally equivalent to identity DMS. The obtained peformance similarities of symbolic and identity DMS are in fact strong presumptive evidence that recognition rather than recall processes are at play in our data and in the monkey work. Within each species and across the two DMS tasks, the maximum delays reached were comparable, error rates were similar, and in this study both symbolic and identity DMS showed the same negative prior-trial effect when samples were changed between trials. This latter comparison, to our knowledge, has not been made for monkeys in symbolic vs. identity DMS tasks. In human memory work, at least, we typically find rather large differences between recall and recognition performance (Kintsch, 1970). Also, recall and recognition are generally differentiated operationally by whether the retrieval cues are (recognition) or are not (recall) uniquely associated with the to-be-remembered information (Watkins, 1979), and there are generally fewer overt retrieval cues available in recall than in recognition tasks (Wickelgren, 1979). Clearly, in symbolic matching there is a unique association between sample and test stimulus, as in identity matching, and the number of retrieval cues available in the two DMS conditions are the same. There seems little basis, therefore, for describing symbolic DMS as other than a recognition memory procedure.

For symbolic and for identity DMS, changing the current-trial sample from its value on the immediately prior trial (i.e., A-B or B-A) resulted in significantly poorer performance than did retaining the sample value (i.e., A-A or B-B). This effect did not hold for probe DMS, however. Instead, errors in probe DMS were related to the value of the probe of the immediately prior trial, and not to the value of the sample. During nonmatching probe trials, in which the current-trial probe and sample were different (i.e., A-b or B-a), there were significantly more errors made when the prior-trial probe was the same as the current-trial probe and, therefore, different from ("nonsupportive" of) the current-trial sample (i.e., a-..-B-a or b-.-A-b). This was true regardless of the support or nonsupport of the prior-trial sample. For matching probe trials (probe same as sample), there were no significant effects of two-trial probe or sample conditions. Indeed, error rates in probe DMS for over 3,000 match trials were a very low $.6 \%$, as compared with $3.7 \%$ for a comparable number of nonmatch trials (Table 3 ). The greater error rate during nonmatch trials may have reflected, in part, a bias toward responding "Yes" ("probe matches sample") under uncertainty, or a bias toward responding to the locus of a sound rather than to a silent speaker, if uncertain. This bias was not present in a later, more complex probe procedure that has already been reported (Thompson \& Herman, 1977), but efforts were made in that later study to retrain response tendencies if bias was detected.

The difference in the locus of interference from the prior trial for probe DMS as compared with either symbolic or identity DMS was not an artifact of the order of testing of these three DMS conditionssymbolic, identity, and probe. Detailed analyses of the effects of changing and retaining samples within each replication of a DMS condition showed a sudden change in relative error trends when the probe condition was reached (Table 4).

How is this set of findings to be integrated into a broader view of proactive interference in DMS? One view is that we may be looking at effects arising at the decision stage of memory. In identity and symbolic DMS, the two test sounds, hypothetically acting as two independent retrieval cues, access two independent memory traces and may force a comparative judgment at the decision stage of memory. The comparisons might be based on the values of "time markers" associated with the two traces, or with a correlate of time, such as strength. In contrast, the single test sound in probe DMS may force an absolute judgment of recency or strength. In human work, it is known that comparative judgments can yield decisions or decision strategies different from those taken in absolute judgments (Helson, 1964; Wickelgren, 1979). The result is that different error patterns may emerge in the two types of judgment situations. Such may have been the case with the different judgments required of the dolphin in the present study.

Another view focuses directly on the temporal discrimination hypothesis (TDH) of DMS performance as advocated most strongly by D'Amato (1973). Consider that the probe of the immediately preceding trial and the sample of the current trial are separated in time by the response latency of the dolphin in making its choice of paddle to press, plus the ITI. In contrast, the sample of the prior trial is separated further in time from the sample of the current trial: that is, by the same durations noted for the probe, plus the 
duration of the prior-trial delay interval. It seems reasonable, therefore, that the probe of the prior trial rather than the sample would exert the major interfering effect on the discrimination of relative recency. In symbolic and identity DMS, this effect does not occur because there are two test sounds (i.e., two competing probes) given in close temporal sequence; their nearly equivalent time "tags" would tend to preclude either from being prepotent. This leaves only the sample of the prior trial, which stands alone as a stimulus, as a source of temporal interference.

Playback of the selected sample sound after a paddle press potentially provides a temporal marker on the playback sound that could produce temporal interference at the succeeding trial. If this were so, the effect could be considerable, since playback was the last occurring discriminably different event of each trial. The only exception was in probe DMS, in which playback was omitted after an incorrect response. That playback had no effect on responses at the succeeding trial was indicated by the results of the analysis of interference in probe DMS. Thus, in correctly selecting the silent speaker in response to a nonmatching probe, the dolphin heard playback of the sample of that trial, which was different from the probe. Yet, as was shown, it was the probe, and not the sample (or by implication, playback of the sample), that influenced current-trial performance. It appears, therefore, that playback acted mainly as a predictor of reward and was not processed and stored as differential temporally tagged information.

In summary, the results for all three DMS conditions demonstrated interference from prior-trial events, although the source of the interference in probe DMS was different from the source in symbolic or identity DMS.

D'Amato (1973) viewed the animal's task in DMS as largely one of discriminating between the relative recency of occurrence of familiar stimuli as samples. He suggested that many of the effects seen in DMS were attributable to interference with or failures of temporal discrimination. The present data, as well as earlier data (Herman, 1975; Worsham, 1975), on the influence of the sample or probe values of the prior trial on current-trial performance is supportive of this hypothesis (also see Thompson \& Herman, 1981, for similar effects in delayed discrimination tasks).

While the temporal discrimination hypothesis (TDH) is attractive as an interpretation of delayed matching results, and temporal discrimination factors undoubtedly influence DMS performance in the cases cited and in others, additional important memorial factors may also be at play in DMS. The work of Grant and Roberts (1976), showing that changing or not changing samples across successive trials does not influence pigeon DMS performance, is a worrisome exception to the hypothesis, restricting its species generality. Grant and Roberts also pointed to un- published work by Worsham and D'Amato on visual DMS in monkeys which showed that manipulations of the intervals between appearance of a visual stimulus as sample accounted for only a relatively small percentage of the error. In the current study, it was noted additionally that increasing the delay interval in the current trial did not systematically increase the magnitude of the interfering effect of changing the sample sound (for symbolic and identity DMS). The current study also failed to find a significant effect of ITI values of $15 \mathrm{vs.} 30 \mathrm{sec}$. In general, shorter ITIs should make for more difficult temporal discriminations (D'Amato, 1973; Herman, 1975). Also, Thompson and Herman (1981) noted that performance was poorer when sample sounds were mapped onto spatial responses in delayed spatial discrimination tasks than when they were mapped onto responses to test sounds as in the current DMS studies. The nature of the response ought not to influence the discrimination of time tags. Finally, in a serial proberecognition DMS procedure reported in Herman (1980), manipulation of the spacing or the rate of presentation of a list of sample sounds did not significantly affect performance. Temporal discriminations ought to be more difficult with closer spacing of successive samples. Undoubtedly, other exceptions could be given (e.g., see Medin, 1980; Nelson \& Wasserman, 1978). Taken together, these various findings suggest that there is more to delayed matching than only temporal discrimination.

Another problem for TDH is that it is difficult to express all forms of the DMS task as primarily problems in temporal discrimination. Different forms of the DMS procedure may tap different dimensions of memory (Herman, 1975), a point that has not always been clearly recognized in the literature. While the use of a small set of highly familiar sounds from which a to-be-remembered sample item is randomly selected at each DMS trial (Herman, 1975; this study) underscores temporal-ordering (nonassociative) processes in memory, the use of an open set of sample sounds not previously heard by the animal, from which a novel sample is chosen at each trial (Herman \& Gordon, 1974), emphasizes associative processes (cf. Wickelgren, 1979). The use of multiple auditory samples in list form (Thompson \& Herman, 1977; Herman, 1980) may tap still additional memory dimensions, such as buffer storage limitations in highinformation-rate tasks or decision strategies taken under uncertainty about item recognition. Emphasis on only one form of DMS task may lead to restricted views of the memory system of the animal or to restricted theories of memory processes. The integration of results from different DMS procedures, and from other types of memory tasks as well, should eventually yield a comprehensive image of memory characteristics, specializations, and constraints in the studied species. 
The difference between the use of a small set of familiar samples and the use of a large or unbounded set of relatively unfamiliar or even novel samples may also be expressed as a difference in the ease of temporal discrimination of recent samples. With large sets, and with the trial-by-trial order of the samples randomly determined, there is, on the average, an increased time between the first occurrence of a sample and its next occurrence, as compared with small sample sets. Hence, there would be a decrease in temporal interference among the samples of the larger set. However, in emphasizing this temporal distinction, one gives short shrift to the considerable associative demands on memory for encoding, storing, and retrieving the qualitative attributes of the many stimuli given. The associative aspects of memory in such cases are neglected in selective emphasis on the nonassociative aspects.

Associative memory may at times be outstanding. For example, in the Herman and Gordon (1974) study, the dolphin Kea made no errors at all $(100 \%$ correct performance) over 169 successive identity DMS problems, each using a novel sample sound. The delays in many cases were $60 \mathrm{sec}$ long and in a few cases $90 \mathrm{sec}$. After one error in six successive DMS trials using 120 -sec delays, Kea self-terminated, as she did in this study. In contrast, in the present paradigms there were at many times $3 \%$ to $10 \%$ error for delays less than $60 \mathrm{sec}$ and as much as $10 \%$ to $15 \%$ error at delays on the order of $90 \mathrm{sec}$. Thompson (Note 1) noted similar competencies in associative memory in four test-naive cynomolgus monkeys given novel visual samples at each identity DMS trial. Learning was rapid and delays reached by the monkeys at levels of $80 \%$ correct or better were in several cases greater than $4 \mathrm{~min}$. This was considerably longer than the delay limits reported by D'Amato (1973) and others for monkeys that are relatively unpracticed and are given visual DMS tasks employing only a few, repeatedly used samples whose occurrence must be temporally discriminated.

Wickelgren (1979) has emphasized that human memory is mainly associative and that stored information is generally organized and accessed by its content (as in the content-addressable memories of some computers). According to Wickelgren, one reason why humans are comparatively poor at judgments of relatively recency is that such judgments are best done through a location-addressable (nonassociative) process, as in a search for information on a tape recorder, rather than through a memory system largely organized in a content-addressable (associative) format. The data on dolphin and monkey showing the comparative weakness of judgments of relative recency as contrasted with remembering nontemporal features suggests that memory in these species may also be principally organized as a content-addressable system.

\section{REFERENCE NOTE}

1. Thompson, R. K. R. Comparisons between visual delayed matching to novel and nonnovel stimuli in monkeys. Paper presented at the meeting of the Western Psychological Association, Sacramento, April 1975.

\section{REFERENCES}

Blough, D. S. Delayed matching in the pigeon. Journal of the Experimental A nalysis of Behavior, 1959, 2, 151-160.

D'AмAто, M. R. Delayed matching and short-term memory in monkeys. In G. H. Bower (Ed.), The psychology of learning and motivation: Advances in research and theory (Vol. 7). New York: Academic Press, 1973.

D'AмAтo, M. R., \& Cox, J. K. Delay of consequences and shortterm memory in monkeys. In D. L. Medin, W. A. Roberts, \& R. T. Davis (Eds.), Processes of animal memory. Hillsdale, N.J: Erlbaum, 1976.

D'Aмato, M. R., \& Worsham, R. W. Retrieval cues and shortterm memory in Capuchin monkeys. Journal of Comparative and Physiological Psychology, 1974, 86, 274-282.

Dewson, J. H., III, \& Burlinane, A. C. Auditory discrimination and recall in monkeys. Science, 1975, 187, 267-268.

GaFFAN, D. Response coding in recall of colors by monkeys. Quarterly Journal of Experimental Psychology, 1977, 29, 587-605.

Grant, D. S., \& Roberts, W. A. Sources of retroactive inhibition in pigeon short-term memory. Journal of Experimental Psychology: Animal Behavior Processes, 1976, 2, 1-16.

Helson, H. Adaptation level theory. New York: Harper \& Row, 1964.

HERMAN, L. M. Interference and auditory short-term memory in the bottlenose dolphin. Animal Learning \& Behavior, 1975, 3, 43-48.

Herman, L. M. Cognitive characteristics of dolphins. In L. M. Herman (Ed.), Cetacean behavior: Mechanisms and functions. New York: Wiley-Interscience, 1980.

Herman, L. M., \& Arbeit, W. R. Frequency discrimination limens in the bottlenose dolphin: 1-70 KC/S. Journal of Auditory Research, 1972, 2, 109-120.

HERMAN, L. M., \& ARBEIT, W. R. Stimulus control and auditory discrimination learning sets in the bottlenose dolphin. Journal of the Experimental Analysis of Behavior, 1973, 19, 379-394.

Herman, L. M., \& Gordon, J. A. Auditory delayed matching in the bottlenose dolphin. Journal of the Experimental Analysis of Behavior, 1974, 21, 19-26.

HonIG, W. K. Studies of working memory in the pigeon. In S. H. Hulse, H. Fowler, \& W. K. Honig (Eds.), Cognitive processes in animal behavior. Hillsdale, N.J: Erlbaum, 1978.

JARRARD, L. E., \& MoIse, S. L. Short-term memory in the monkey. In L. E. Jarrard (Ed.), Cognitive processes of nonhuman primates. New York: Academic Press, 1971.

Johnson, C. S. Sound detection thresholds in marine mammals. In W. N. Tavolga (Ed.), Marine bioacoustics (Vol. 2). New York: Pergamon, 1967.

KEPPEL, G. Design and analysis: $A$ researcher's handbook. Englewood Cliffs, N.J: Prentice-Hall, 1973.

KINтsCH, W. Learning, memory and conceptual processes. New York: Wiley, 1970.

Maki, W. S., JR., Moe, J. C., \& Bierley, C. M. Short-term memory for stimuli, responses, and reinforcers. Journal of Experimental Psychology: Animal Behavior Processes, 1977, 3, 156-177.

Mason, M., \& Wilson, M. Temporal differentiation and recognition memory for visual stimuli in rhesus monkeys. Journal of Experimental Psychology, 1974, 103, 383-390.

Medin, D. L. Proactive interference in monkeys: Delay and intersample interval effects are noncomparable. Animal Learning \& Behavior, 1980, 8, 553-560.

Nelson, K. R., \& Wasserman, E. A. Temporal factors influencing the pigeon's successive matching-to-sample perfor- 
mance: Sample duration, intertrial interval, and retention interval. Journal of the Experimental Analysis of Behavior, $1978,30,153-162$.

Robents, W. A., \& Grant, D. S. Studies of short-term memory in the pigeon using the delayed matching-to-sample procedure. In R. T. Davis, D. L. Medin, \& W. A. Roberts (Eds.), Processes of animal memory. Hillsdale, N.J: Erlbaum, 1976.

RoITBLAT, H. L. Codes and coding processes in pigeon short-term memory. Animal Learning \& Behavior, 1980, 8, 341-351.

Thompson, R. K. R., \& Herman, L. M. Underwater frequency discrimination in the bottlenosed dolphin $(1-140 \mathrm{kHz})$ and the human (1-8 kHz). Journal of the Acoustical Society of America, 1975, 57, 943-948.

Thompson, R. K. R., \& Herman, L. M. Memory for lists of sounds by the bottlenosed dolphin: Convergence of memory processes with humans? Science, 1977, 195, 501-503.

Thompson, R. K. R., \& Herman, L. M. Auditory delayed discriminations by the dolphin: Nonequivalence with delayed matching performance. Animal Learning \& Behavior, 1981, 9, 9-15.

WatKIns, M. J. Engrams as cuegrams and forgetting as cue overload: A cueing approach to the structure of memory. In C. R. Puff (Ed.), Memory organization and structure. New York: Academic Press, 1979.
Wickelgren, W. A. Cognitive Psychology. Englewood Cliffs, N.J: Prentice-Hall, 1979.

WorshaM, R. W. Temporal discrimination factors in the delayedmatching-to-sample task in monkeys. Animal Learning \& Behavior, 1975, 3, 93-97.

Yunker, M. P., \& Herman, L. M: Discrimination of auditory temporal differences by the bottlenose dolphin and by the human. Journal of the Acoustical Society of America, 1974, 56, 18701875.

\section{NOTE}

1. To control for experimentwise error rate in these multiple individual comparisons, the level of significance, $a^{\prime}$, was adjusted using Dunn's procedure (Keppel, 1973), where $a^{\prime}=a / k$ and $a$ is the initial alpha chosen (.05), and $\mathrm{k}$ is the number of comparisons. For the ITI effect, $\mathbf{k}=\mathbf{3}$ and $\mathrm{a}^{\prime}=\mathbf{0 1 7}$.

(Manuscript received October 27, 1980; revision accepted for publication July 4, 1981.) 